\title{
Making Web Sites Be Places for Social Interaction
}

\author{
Andreas Girgensohn \\ FX Palo Alto Laboratory, Inc. \\ 3400 Hillview Avenue, Bldg. 4 \\ Palo Alto, CA 94304, USA \\ andreasg@fxpal.com
}

\author{
Alison Lee \\ IBM TJ Watson Research Center \\ 19 Skyline Drive \\ Hawthorne, NY 10532, USA \\ alisonl@us.ibm.com
}

\begin{abstract}
Technology can play an important role in enabling people to interact with each other. The Web is one such technology with the affordances for sharing information and for connecting people to people. In this paper, we describe the design of two social interaction Web sites for two different social groups. We review several related efforts to provide principles for creating social interaction environments and describe the specific principles that guided our design. To examine the effectiveness of the two sites, we analyze the usage data. Finally, we discuss approaches for encouraging participation and lessons learned.
\end{abstract}

\section{Keywords}

Awareness, common ground, communication, cooperation, participation, practice and experience, social interaction, usage data, usability, Web-based collaboration.

\section{INTRODUCTION}

Developments in information and communication technologies have increased the opportunities and means for coupling technology with certain social practices (e.g., town hall meetings, support networks) that would influence people's ability to act together. In addition to the immediate outcome from such technology-mediated interactions, Resnick [21] has suggested that, as a side effect, such interactions could create productive resources - socio-technical capital $^{l}$. The capital may consist of artifacts created from the interactions or relationships and practices developed through repeated social interactions. Such capital can enable future social interactions. Use of the capital by a group to act collectively may not simply consume the resource but can reproduce more of it.

The Web is one such social technology. It enables people to share experiences, to expose tacit knowledge, to make recommendations, and to discuss a wide range of topics [22, $26,27]$. It also enables people to get to know other people and to seek out affiliation, companionship, and support [5, $17,16,22]$. The Web enables people to work together, to conduct economic transactions, and businesses to build personalized relationships with their customers [13, 15]. Many of these social by-products are achieved by leveraging affordances of the technology for information dissemination, communication, support, self-service transactions, and mediation. The combination of these Web functions and

Permission to make digital or hard copies of all or part of this work for personal or classroom use is granted without fee provided that copies are not made or distributed for profit or commercial advantage and that copies bear this notice and the full citation on the first page. To copy otherwise, or republish, to post on servers or to redistribute to lists, requires prior specific permission and/or a fee.

CSCW'02, November 16-20, 2002, New Orleans, Louisiana, USA.

Copyright 2002 ACM 1-58113-560-2/02/0011...\$5.00. social processes can jointly influence the ability of people to act together in productive ways such as the creation of socio-technical capital [21].

Digital cities [9], community networks [24], portals such as eBay and Slashdot, and older technologies based on electronic bulletin board systems, newsgroups and mailing lists $[22,23]$ are just some of the examples of social interaction spaces. This interest in social interaction spaces is not limited to work contexts but extends to commercial and noncommercial contexts involving large and small groups of non-technical end-users $[1,5,10,22]$. For each successful site, there are many more that fail for lack of interest, participation, usability, and sociability. Design principles for creating such social interaction sites are beginning to emerge $[11,12,15,20]$ but there is a scarcity of case studies of designs applying these principles or analyses of the effectiveness of designs.

In this paper, we present a case study of the design of two social interaction Web sites: CHIplace and Portkey. In the next section, we briefly characterize the target user groups of these two social interaction sites. We then review several related efforts to provide principles for the design of social interaction environments. We describe the specific principles that guided our designs and how they were instantiated in a technical solution. We present an analysis of the patterns of use to examine the effectiveness of the Web-site designs for facilitating social interactions. In conclusion, we discuss the design implications of our findings.

\section{TWO TARGET SOCIAL GROUPS}

Both Web sites described in this paper had the goal of fostering interactions among participants. The first Web site, CHIplace, was developed for the ACM CHI 2002 conference to extend the interaction opportunities and the interactions among people both in time and space. The second site, Portkey, was developed for the summer interns at IBM TJ Watson Research Center to enable the interns to exchange helpful information and experiences and to develop the social networks - personal and professional - that they needed to function effectively in their new environment. While both projects shared similar design goals for participation, social interaction, collective action, and development of social networks, they had different requirements and interactional constraints. For example, the CHIplace group was expected to be large and diverse whereas the Portkey group was fixed and characterizable. CHIplace was

\footnotetext{
${ }^{1}$ Socio-technical capital is a resource produced as a side effect of technology-mediated social interaction. It can be accumulated and made available for people to create value for themselves or others [21]
} 
designed to be an open-access system while Portkey was restricted to registered users; the interns, researchers, and staff involved in the 2001 summer internship program.

\section{CHIplace Users}

Of the 1176 CHIplace users that were registered at the end of April 2002, 49 were CHI conference committee members. Many unregistered users visited CHIplace but we have no information about them. Among those who provided country information, $52 \%$ were from the US and $48 \%$ from 44 non-US countries. The most popular of the 11 roles that people could select from were researcher $(44 \%)$, designer (26\%), student (22\%), consultant (18\%), and interface evaluator $(17 \%)$.

\section{Portkey Users}

Roughly half of the Portkey members (738) were summer interns (343) who were responsible for 95\% of the site usage. The summer interns came from 130 different universities located in 13 countries. Most students came from universities in the US (84\%), France (4\%), and Germany (3\%). Their backgrounds were in computer science $(48 \%)$, engineering (27\%), sciences (11\%), business $(5 \%)$, mathematics (3\%), and other disciplines (6\%). Among the students who indicated their country of origin, $43 \%$ were from the US and $57 \%$ from non-US countries. 54\% of the students were enrolled in a Ph.D. program, $15 \%$ in a Masters program, $29 \%$ in a Bachelors program, and 1\% in high school. These demographics illustrate the diversity and international makeup of the students.

\section{DESIGN PRINCIPLES FOR A SOCIAL WEB SITE}

How might one build Web sites that have a focus on people and social interactions? Kim [11], Kollock [12], and Preece [20] have independently presented three related sets of design principles and strategies. Kim's nine design strategies provide the most systems-oriented approach of the three [11]. Her strategies cover purpose, people, gathering places, evolving roles, leadership, rules and policies, planned events, rituals, and support of sub-groups as well as a variety of technologies for each strategy. She also presents three underlying design principles: a) design for growth and change, b) create and maintain feedback loops, and c) empower members over time. Taken together, the nine strategies and three design principles provide a technology-facilitated social-scaffolding approach to social organization.

Kim's strategies overlap extensively with Preece's two design principles: a) designing for usability and b) supporting sociability [20]. The first design principle focuses on good usability so that people can interact and perform tasks easily and effectively. The second design principle focuses on social interaction and three components of sociability: a) purpose, b) people and roles, and c) policies related to governance, membership, codes of conduct, privacy, security, and copyright protection. Preece also examines software selection options and technology development approaches. Her principles draw from an HCI perspective of understanding individuals, groups and their "work" environments in the design and development of such systems.

Finally, Kollock's proposal focuses on the key sociological challenges with building online communities [12]. He argues that technological and user interface challenges exist, but the sociological challenges are greater because of the poor understanding of how they impact a successful online community. His design principles are derived from work on cooperation and social dilemmas and focus on fostering social interaction, cooperation, collective action and social order $[2,19]$. The implications of his proposal do not differ radically from Kim [11] or Preece [20] but are more grounded in a sociological perspective. His principles emphasize the need for a) repeated social interaction, b) a persistent identity built on information about the person and their behavior until now, c) well-defined group boundaries, d) the evolution of norms and rules regarding collective resources and e) a means to monitor and sanction member's behavior.

\section{CHIplace and Portkey: Key Design Challenges}

The designs of our two sites were, first and foremost, driven by the need to identify the key sociological challenges, of which there were three [12]. "Encouraging user participation" is invariably an important goal and a key challenge in any socio-technical system. Without it, the people, their presence, and their activities which define the social context would not exist, social interaction would not be possible, and social organization would not emerge [25]. This challenge was made even harder because both projects had scarce human resources for creating and evolving content and for leading and breathing life into the social system.

"Fostering social interactions" is a key focus of all three sets of design principles. Kim discusses the importance of bringing people together and the importance of scheduled events in reinforcing a sense of belonging. One of Preece's two design principles focuses on supporting social interaction. Kollock points to the importance of two individuals meeting again in the future as a condition for cooperative relationships to emerge and persist. Repeated social interactions are important for increasing familiarity, developing relationships, reminding people about what they have in common, strengthening ties to an organization and helping to develop a positive regard for the social system $[2,14]$.

"Promoting visibility of people and their activities" is a key challenge because, without people, there is no community $[11,20]$. In making them visible, a number of important issues can be addressed. First, Kollock [12] points out that a persistent and up-to-date identity is an important condition for cooperation both in letting individuals identify each other and for knowing how people behaved in the past. It is also a crucial element for any community governance system to operate in which people need to be able to identify people, to monitor inappropriate behavior, and to hold accountable those who misbehave. Second, people provide help to and interact with people they know, people they like, people who are similar, and people who have helped them [4]. Therefore, it can help "people to get to know each other." Third, theories of pro-social motivation suggest that people provide help to strangers even when this help is personally costly [4]. The reasons are that helping others can increase self-esteem, personal identification with organization, stature within the organization, feelings of commitment, helping to strengthen the social organization, and 


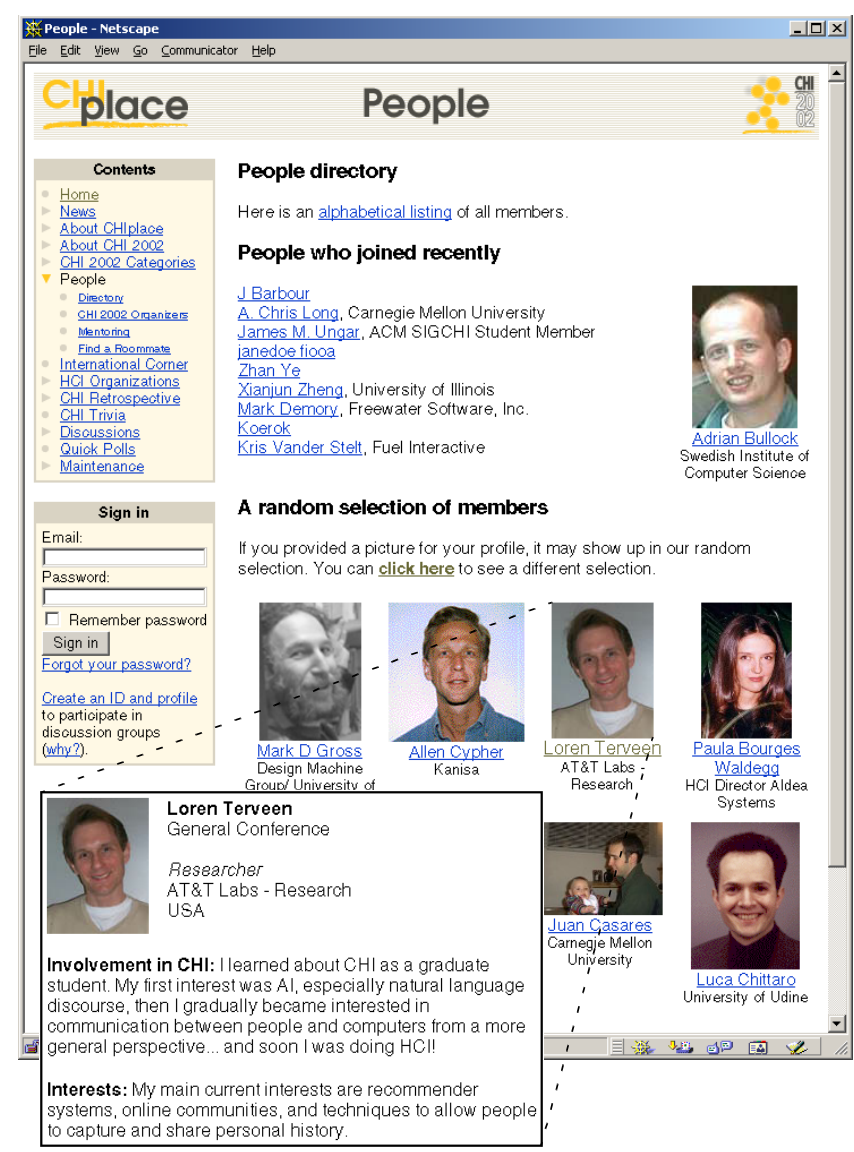

Figure 1: People page on CHIplace with member profile.

helping to promote generalized reciprocity. Therefore, attributing and recognizing the kindness and efforts of individuals can help motivate people to help others. Finally, promoting the visibility of people and their activities can encourage people to become active and to prolong participation. Related work with positive reputation systems have shown the value of a good reputation in economic transactions and in preventing people from defecting [13].

Aside from the three key sociological challenges, the design of our two social interaction Web sites had two additional design challenges. The first was "designing for usability." Clearly, if the system did not have good usability, it would raise the bar for participation. Also, given the challenges with motivating people to participate, bad user experiences could turn away many potential participants.

Our second design challenge was "maintaining the site with minimal resources." Both projects had very few human resources; CHIplace was a community service by the two principals and Portkey was largely a two-person research project. This meant that we needed to rely on using technology to help with the management of the sites. Wherever possible, the Web sites needed to grow and evolve through the contributions of their participants.

\section{DESIGN OF TWO WEB SITES}

We provide some examples of and some approaches used to address the five key design challenges for CHIplace and Portkey. A seeding, evolutionary growth, and reseeding

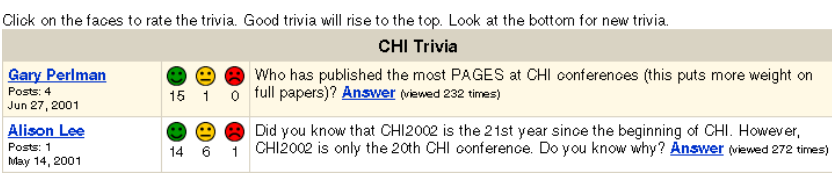

Figure 2: CHIplace trivia

approach was applied [7]. Periodic analyses (formal and informal) were conducted to identify how the sites were evolving and how socio-technical solutions could facilitate and nurture their evolutionary growth.

\section{Promoting Visibility of People and Their Activities}

Both sites provided a number of mechanisms that enabled participants to construct and evolve a persistent and verifiable identity. First, each registered participant had a profile containing known and user contributed information that was presented through individual people pages. Various options were considered in the design of the profile; real-life details about the person, a verifiable pseudonym as in eBay; or registered anonymity as in MOOSE Crossing [3]. The real-life option was chosen in both cases because people were expected to meet and interact both online and face-to-face. Because Portkey was a closed community, all profiles were created by the administrators. CHIplace profiles required a valid email address to which the password was sent.

Profiles contained real-life information about each person including their name, photograph, home country, email address, and a link to a Web page with more information (see Figure 1). The CHIplace people profile also contained people's affiliation, their HCI role (e.g., practitioner), possible role and goals for CHI 2002, their HCI interests, and how they became involved in HCI. Portkey maintained additional information for students and for permanent researchers. This included information about their technical and outside interests and their research project. Portkey users were also asked to provide up to five questions that other people could ask them. These questions were intended to help Portkey participants initiate conversations when they met face-to-face. In addition, the student's profile included their discipline, university and the researchers who made up their management chain.

Information about a person's activities and behaviors on the site, known as traces, is an important element of a persistent and up-to-date identity. This includes explicit and implicit actions that identify both the individual's interests and other people's interests. A clear illustration of traces can be found in the CHIplace trivia (see Figure 2). Each trivia provides an author attribution as well as an indication of how many they have authored. This recognizes a person's participation and level of participation. Second, each trivia has an answer component. The number of times the answer is viewed provides an implicit indication of interest in the question. Finally, people can explicitly cast votes about what they think of the trivia. The number of positive, neutral, and negative votes reflects interest in the answer. This series of attributions empower authors and readers by recognizing the author's efforts and the readers' interest. Furthermore, such traces provide insights to potential trivia authors for what interests the site participants and how to construct a trivia. 


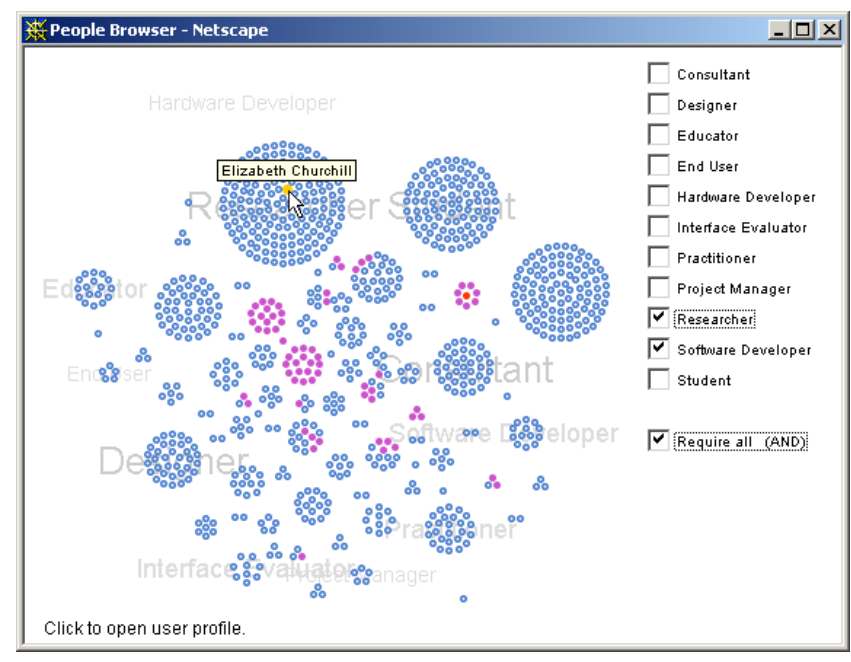

Figure 3: People Browser.

Finally, both Web sites provided a number of social browsing tools that enabled the participants to get to know the other participants of the site.

- A list of people that joined most recently together with a randomly selected picture of one of those people (see the top of Figure 1). This publicly recognized new members and provided a technology-facilitated means of introducing new members to the community [11].

- A gallery of eight randomly selected pictures of members (see the bottom of Figure 1). For Portkey, four of the pictures were interns and four were permanent researchers. This used serendipity to acquaint people to other participants at the Web site.

- People directories sorted by last name or country. On Portkey, additional directories sorted by such attributes as first name, discipline, university, and technical and outside interests were provided. These directories provided a means for people to learn about co-participants that shared similar attributes.

- CHIplace also provided a lightweight people browser that visually grouped the CHIplace participants with similar HCI roles (see Figure 3). Each member was represented as a dot. Rolling over the dot revealed the name of the person and clicking on the dot led the user to the person's profile. Users could also select members by their roles.

\section{Encouraging User Participation}

We created awareness about the sites to drive traffic to them. Invitations to participate were sent to various email distributions and groups. We also registered the CHIplace site with various search engines and placed links at ACM Web sites. Those links were moderately effective as $11 \%$ of the outside CHIplace page accesses were referred by other sites. Search engines accounted for much of the referred traffic (71\%) and the ACM Web sites for much of the rest (15\%).

To sustain awareness, we kept participants apprised of new additions and ongoing developments on the site. The home pages of both sites devoted areas for presenting this information (see Figure 4). Many of the participants were discretionary users both in terms of goals and motivation for

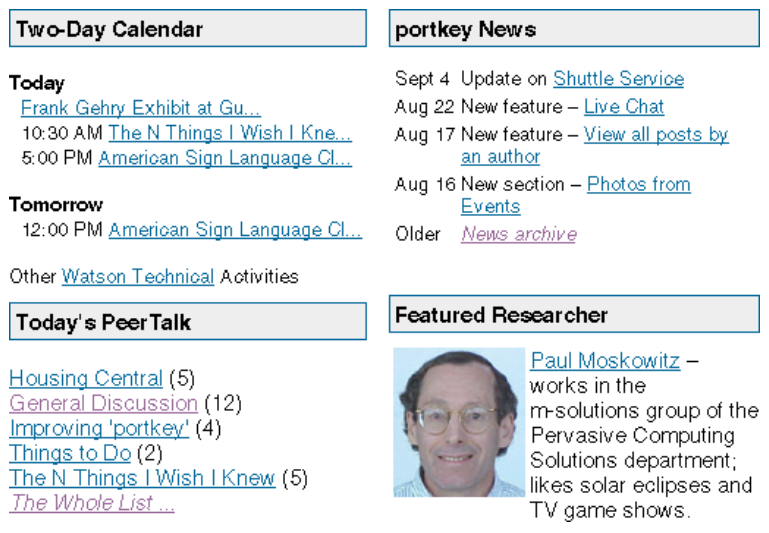

Figure 4: Part of the Portkey home page providing a calendar of events, news and discussion.

participation. This turned out to be particularly true for many of the CHIplace participants and Portkey permanent researchers. In recognition of this, we sent out periodic newsletters via email that summarized new additions and ongoing developments.

Both sites had an incentive-based participation reward program. For example, Portkey had monthly contests where an eligible intern would be randomly drawn as a winner of a $\$ 1000$ gift certificate. To qualify for the monthly drawing, the intern needed to provide a completed profile and to make a minimum of three postings to the Portkey discussion forums. Then, additional posts gave the eligible intern additional ballots in the drawing. This was successful in motivating people to fill out their profiles (174), to provide a photo (128), and to participate in discussion forums (144 students made at least one posting). However, this was not the sole reason for participation as only 73 of the 343 students ever met the eligibility criteria and no more than 47 people in any given month were eligible for the contest.

On CHIplace, users were not required to be registered to view the site's content. We believed that CHIplace participants would benefit if they registered as it would help to create greater awareness of people interested in $\mathrm{CHI}$ and their activities and to build a sense of community on CHIplace. To encourage registration, parts of the site such as the archive of old stories and articles and CHI 2002 full papers were only accessible to registered users.

Recognizing, identifying and attributing participation has been shown to be valuable for encouraging participation [4, $11,13]$. In particular, traces can be instrumental for user participation $[10,12]$. Aside from the trivia example, we provided information such as page views, most viewed, and links on people's profiles, if applicable, to the number of and the list of their authored posts. We only presented such information selectively because limited resources prevented making this pervasive, but we believe that such information can both create awareness of interest as well as recognize participation.

\section{Supporting Sociability: Social Interaction Components}

There has been extensive CSCW research on supporting interaction among geographically distributed co-workers [6]. We recently distilled the research results into a set of 


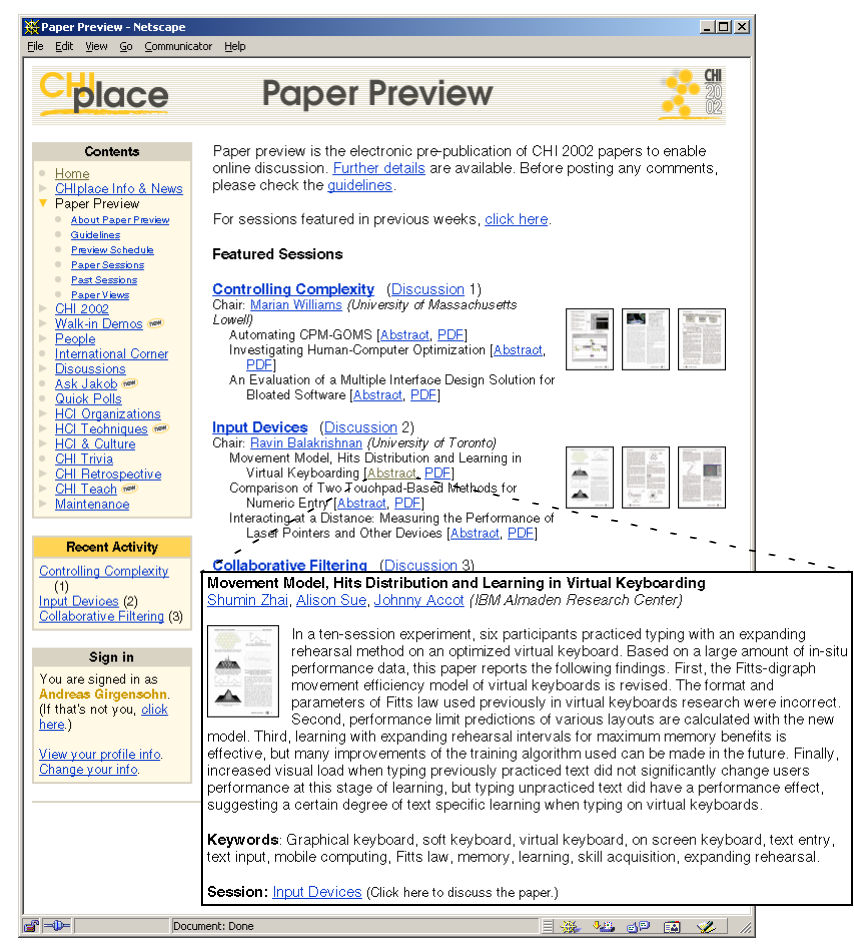

Figure 5: Paper Preview.

requirements and four basic social interface components that can foster social interactions: common ground, awareness, interaction enablers and mechanisms, and place-making for building social interaction sites [15]. We briefly describe each component and provide examples of what these were in CHIplace and Portkey.

\section{Common Ground}

Common ground is the shared understanding among collaborators that permits interactions to proceed smoothly and the shared context for the social interaction. To facilitate the development of common ground among many of the participants who were strangers to each other, they were asked to create profiles. All authored site content was annotated with links to those profiles. Several topical content pages incorporated discussion forums to enable participants to share and discuss issues with other participants.

In addition, we seeded content in areas that represented a common need of the participants. For example, on CHIplace, CHI retrospective consisted of folklore and trivia about CHI's history and a list of past $\mathrm{CHI}$ conferences, past conference chairs, and locations. This provided a context about CHI for newcomers. Another example is the electronic prepublication of CHI 2002 conference papers for discussion (see Figure 5). The aim was to leverage the shared interest that CHIplace members had to help shape the authors' presentations and the moderator's remarks at the conference. On Portkey, we used the official information developed by the human resources group about the program to seed the content of Portkey and supplemented it with "tips" provided by summer interns from the previous year.

The lack of a common or prior history among Portkey members meant that many of the community content opportunities (e.g., trivia) that CHIplace had did not exist on Portkey.
Instead, we solicited material to post on the site. For example, photos from social outings and photos, abstracts and snippets of student poster presentations organized by the departments. We featured researchers at the corporation on a weekly basis on the home page to help make contact with the broader community (see bottom right of Figure 4).

\section{Awareness}

Awareness is the state of knowing or being informed that enables collaborators to interpret others' actions and to guide one's own actions. It consists of a combination of people, activity and contextual awareness.

In addition to people profiles, activity traces, and annotations of content with links to people profiles and activity traces, participants were made aware of new contributions. We provided listings of what's changed to support return visitors. All discussion threads were displayed in chronological order with the most recent at the top. As well, new threads and messages since the last time the user visited were highlighted with a different color. On Portkey, the home page included a section on today's active forums and activities scheduled today and tomorrow (see Figure 4).

\section{Social Interaction Mechanisms}

Collaborators need both the enablers and the tools for social interactions that allow them to act and interact. The enablers provide cues of the availability and variety of social interaction options. The social interaction tools support the social and production-oriented aspects of interactions [14], the synchronous and asynchronous elements of interactions, and the ad-hoc and planned nature of interactions.

On both sites, we used quick polls to engage the audience and to determine the course of action for controversial issues such as the contest on Portkey. These polls provide a a low-effort interaction mechanism that allows users to provide their opinions on a topic without having to cross the hurdle of participating in a discussion forum. In that sense, quick polls can ease users into taking a more active role.

Both sites had discussion forums for different topics. On CHIplace, many of the discussion forums were attached to specific pages. This allows the users to be engaged within the context of what they are viewing to make suggestions or to comment on stories. On Portkey, forums were organized in several categories such as announcements, tips from peers, and social and technical discussion forums.

CHIplace also featured two other social interaction mechanisms: a) writing and voting of trivia (Figure 2) and b) paper preview (Figure 5). These were efforts to engage the participants in discussion, in sharing experiences, and in providing feedback about the value of people's contributions.

\section{Place-making}

Place-making is the cultural and social understanding of the norms and practices appropriate to an interaction setting that emerges over time and through users adapting and appropriating the setting $[8,11,12,20]$.

People profiles are examples of resources that had no restricted or prescribed use. They were free-formed to provide flexibility in the kind of profile information people could provide. There is quite a variety in how people used 


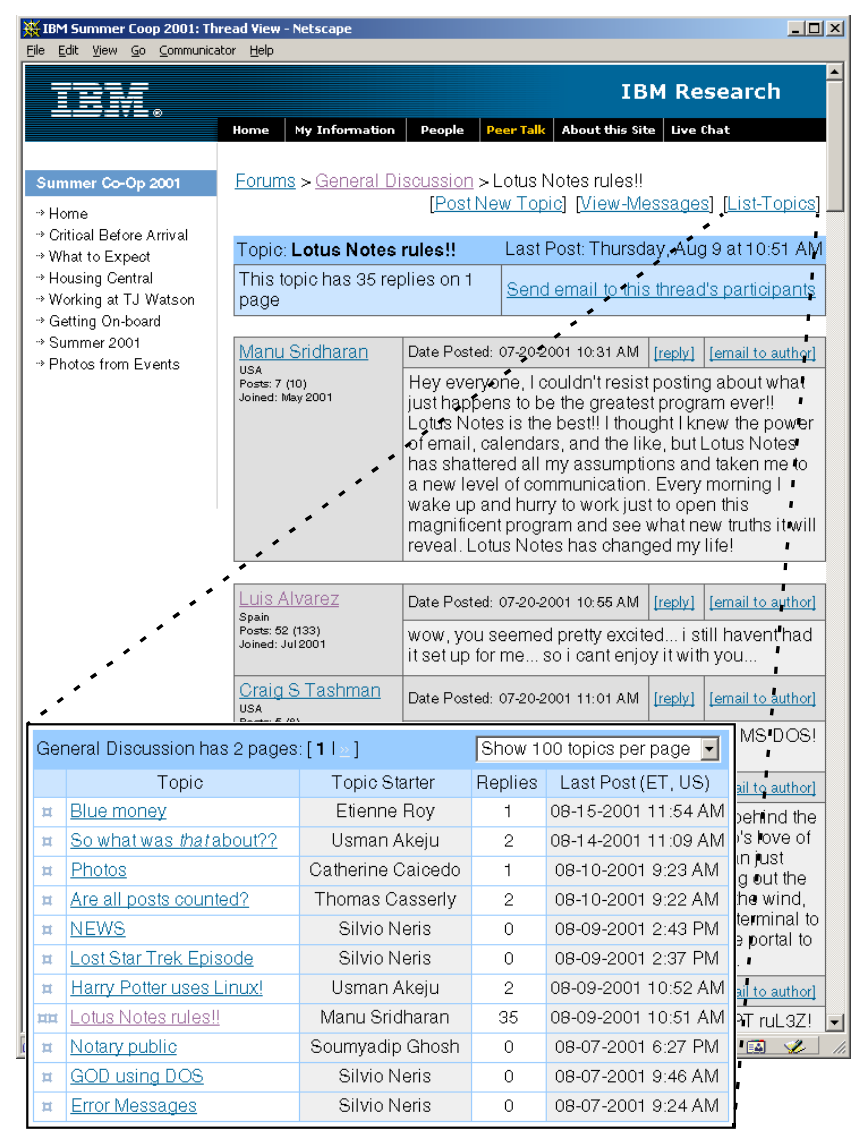

Figure 6: Portkey discussion thread with topic list.

the fields to express information about themselves. As an illustration, some Portkey users chose to provide serious, work-related "Ask Me" questions while others shared their interests or aspects of their personality.

Much more of a sense of place developed on Portkey than on CHIplace. One good example is the behavior of people posting to discussion forums. The contest provided an incentive for people to post many messages. Some of the student interns felt that others abused the system and attempted to demonstrate the absurdity of the situation by starting a nonsensical discussion thread on the virtues of Lotus Notes (see Figure 6). This led to a more serious discussion on proper behavior on the site. Another example is the use of discussion forums for planning social activities such as meeting in the park on the weekend. These episodes illustrate that the discussion forums were more than simply a vehicle for information sharing. They became a vehicle for coordinating get-togethers and for teaching, discussing, and formulating an initial set of norms for using the Web site.

\section{Designing for Usability}

In an effort to keep the barriers to participation low, both sites provided a simple customized form for their users to create and update their profile, a self-service mechanism to reset forgotten passwords and email them a new password, and to upload their photo for their profile. Some pages were customized to display group-specific information to prevent irrelevant information from being presented.
Also, we made the navigation structure visible on each page and positioned it in a consistent location. People and list of discussion forums were always accessible to the participants. On Portkey we also used a timeline to structure the official content about the corporation, the geographic area, work-related topics, and social activities. This allowed the students to access the collection of information of concern to them that was dependent on what phase of the internship they were at (i.e., before arrival, first week of internship, during internship, last week of internship). This design grew out of early fieldwork that revealed that students needed to access different information depending on what stage they were at in the internship.

Browsing of the people pages and people directories was very popular on both sites. People directories facilitated easy browsing of the people profiles by a variety of attributes. On CHIplace, we developed the people browser (see Figure 3 ) to provide the more discretionary users with a quick means of finding out who else was like them and who other CHIplace members were.

\section{Maintaining the Site with Minimal Resources}

All pages on both sites were dynamically generated with JavaServer Pages and a MySQL database. The use of templates and a database simplified adding new pages or modifying existing pages. This approach also allowed us to provide custom information for signed-in users.

We used an open-source Web usage analysis tool called Webalyzer to generate daily and monthly traffic pattern reports. The reports enabled us to note unusual traffic patterns and to examine root causes for such patterns. For example, we found heavy traffic at CHIplace on a number of instances because of referrals by various people.

We developed several scripts that identified problems in Web usage logs that were encountered by site visitors. For instance, connection problems to our site, broken links that we created or that our participants provided and problems with our dynamically generated pages were brought to our attention through these daily generated reports. We were able to exploit Web crawlers that periodically crawled over the site to identify similar problems.

We created scripts that ran frequently to scan for new photos provided by participants. These scripts resized the photos to a recommended size so that our people gallery would not be populated with highly varying sized photos. Also, the scripts cached externally-linked photos to enable our sites to be buffered against vagaries of download and connection problems. Scripts were also developed to provide scheduled notification such as reminders to authors and moderators about the imminent preview of their sessions.

Finally, we did not want to be involved in moderating contributions. Therefore, we decided to only let registered users contribute and to provide author attributions to all contributions. This allowed the sites to use a combination of selfmoderation and community moderation schemes. The latter was particularly instrumental on Portkey when a few individuals tried to post numerous, low-value posts. It led to a huge discussion about appropriate netiquette. 


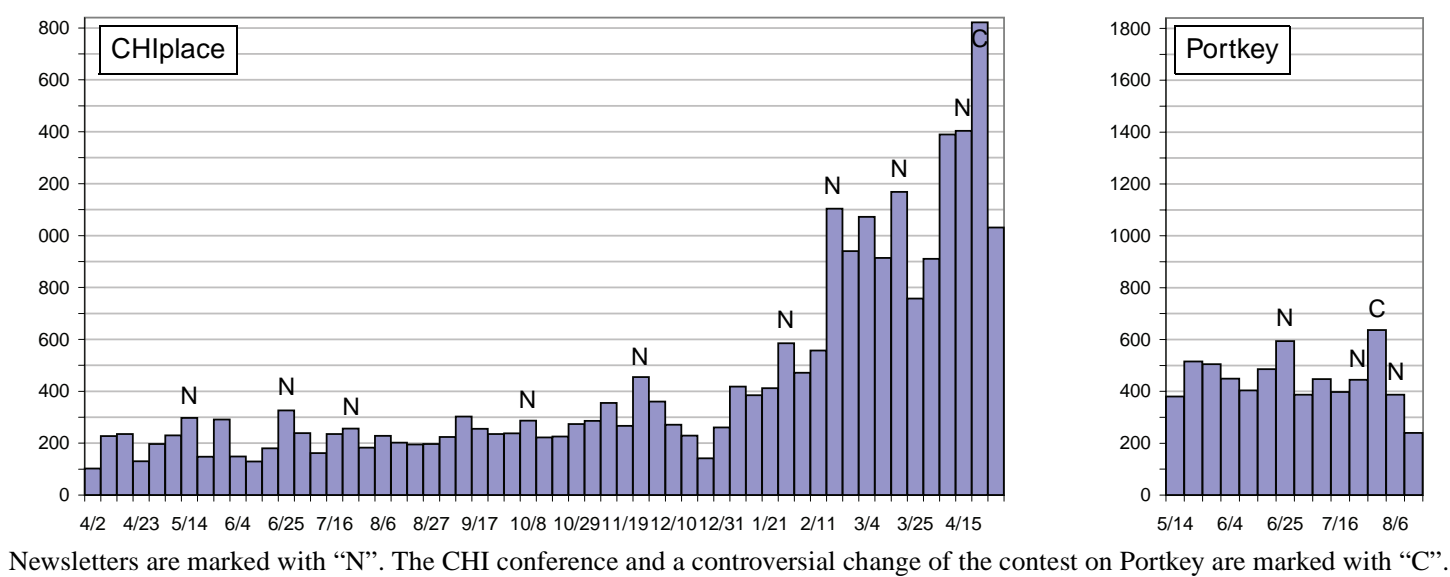

Figure 7: Weekly number of sessions.

\section{USAGE DATA FOR THE WEB SITES}

We analyzed the usage data of the sites to assess the effectiveness of their designs. The data shows interesting trends in response to the introduction of new features and to email notifications sent to various groups. The data also highlights similarities and differences between the two sites.

To find usage patterns, we grouped access to pages into sessions. For our sites, sessions provide a fairly accurate estimate for the number of unique users per day because very few people visit more than once per day judging from the client IP addresses. We also classified pages into meaningful categories and used those categories to show transitions.

\section{How the Data Was Collected}

We collected usage data using a Web server access log that included cookie information. With cookies we could associate page accesses with the servlet sessions maintained by the Web application server. We also kept a sign-in log that was synchronized with the Web server $\log$ via the session identifier.

We only considered successful page accesses (and not accesses to images and other auxiliary files). We also filtered out page accesses that did not come from real users. On Portkey where all users were required to sign in, we ignored all page accesses that could not be associated with an entry in the sign-in $\log$ and filtered out the entries associated with site administrators. Search engine accesses were ignored automatically because there were no sign-ins.

Because sign-in was optional on CHIplace, we used a different approach for filtering out pages. First, we ignored access from the automatic display at the conference. We also ignored access from a set of IP addresses that were used by the site administrators. Unfortunately, that also filtered out some entries by genuine users because one of those addresses belonged to a "socks" server used by company employees. Second, we filtered out all requests from user agents that appeared to be search engine spiders. Finally, we filtered out requests from browsers that did not return the session identifier cookie because we could not properly track those sessions. This last step caused the loss of about $6 \%$ of the usage data on CHIplace. However, as an added benefit, any remaining spider accesses were filtered out because spiders do not return cookies.

\section{Weekly Usage}

We tracked the usage of CHIplace and Portkey for 57 and 14 weeks, respectively. For CHIplace, we had 24,768 sessions (62 per day) and 193,294 page accesses (excluding images and other auxiliary files) and for Portkey we had 6,640 sessions (68 per day) and 155,530 page accesses. Figure 7 plots the weekly number of sessions over time. On Portkey, 95\% of all page accesses were initiated by the students.

When comparing the number of sessions over time, one can see that the usage is fairly constant on Portkey with the exception of the last week when many of the interns had left. Except for the last 11 weeks on CHIplace, Portkey had more than twice as many sessions. Only the start of the paper preview on February 18 pushed the weekly number of sessions above those of Portkey's.

On average, Portkey sessions contain three times as many page accesses as CHIplace sessions. This is mostly due to the heavy use of discussion forums that is discussed later in this section. Figure 8 shows that the average number of pages per session stayed fairly constant on Portkey with a drop in the last two weeks when the summer interns started leaving and not much discussion took place. In contrast, on CHIplace there was a noticeable downward trend during the first four months before leveling off. We believe that this is due to repeat visitors that found only few new pages and

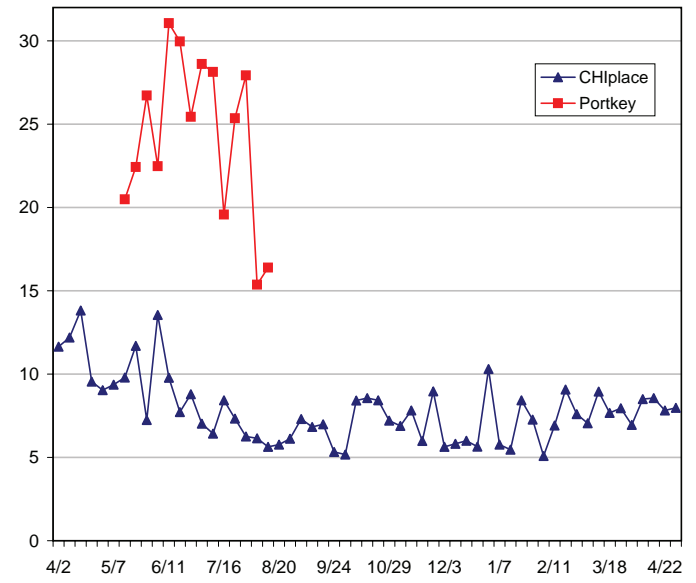

Figure 8: Weekly average of page requests per session. 


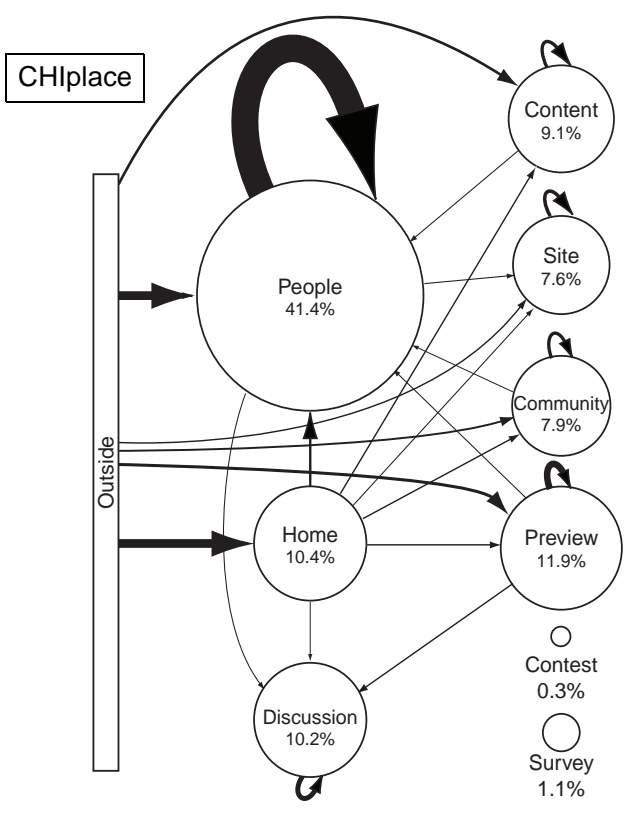

The area of the circles is proportional to the percentage of usage and the thickness of the arrows is proportional to the percentage of transitions. Transitions below $0.7 \%$ are omitted for the sake of clarity.

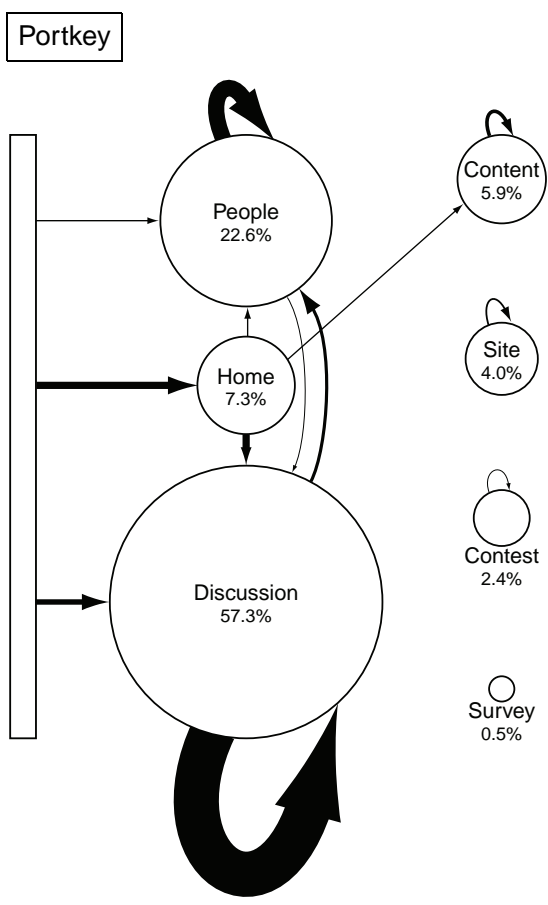

Figure 9: Page category usage and transitions.

much less activity in the CHIplace discussion forums. Interestingly, the introduction of new features such as the paper preview only increased the number of sessions per week and not the number of page requests per session.

Email announcements to participants and other interested people caused spikes in usage on the day of the message. For CHIplace, we sent nine newsletters to the members who requested announcements (see the weeks marked with "N" in Figure 7). Some spikes are noticeable in the weekly view but the effect is much more pronounced on the day of the message. On those days, the number of sessions was on average $60 \%$ higher than on the previous business day and the number of page requests was $86 \%$ higher. Announcements are an effective means to provide awareness of the site existence and new developments. Messages to mailing lists such as all SIGCHI members and CHI announcements proved to be good for drawing in new visitors.

\section{Patterns of Use}

To track patterns of use, we grouped pages into major meaningful categories. While different categorizations of pages are possible, we believe that the chosen categories represent the different types of use well.

- Home. The entry page into the site.

- People.The main people page with random pictures, the people directories, and the individual user profiles.

- Discussion. Pages related to discussion forums, threads, and messages, and pages for posting messages.

- Content. The content specific to the site. On CHIplace, the content includes CHI 2002 conference information. On Portkey, the content includes information about the organization and the intern program.
- Site. Information about the site (e.g., site map, about or help pages) and tools for manipulating user profiles.

- Community. Information provided by the user community. This category exists only on CHIplace and includes stories, articles, organizational overviews, and trivia.

- Preview. Advance information about conference events such as the paper preview and the demo sign-up.

- Survey. User surveys and polls.

- Contest. Information about contests on the two sites.

We used the categories to illustrate usage of each category, to summarize overall usage, and to track transitions between categories (see Figure 9). The graphs are an adaptation of the graph type described by Olson et al. [18].

\section{Discussion Forum Usage}

When comparing the two graphs, we immediately notice the dominant use of discussion forums on Portkey; 144 people wrote 1603 messages (16.4 per day). On CHIplace, the discussion forums were not used much in the 46 weeks before the paper preview started. More than three quarters of the messages were posted during the last 11 weeks and almost two thirds of the discussion activity occurred during that time. In the first 46 weeks, 43 people wrote 58 messages (0.2 messages/day) while 89 people wrote 185 messages (2.4 messages/day) in the remaining 11 weeks.

We believe that the much higher use of the discussion forums on Portkey is due to two reasons. First, the chances to win in a contest of Portkey were increased by posting messages. Even for the people who were not motivated by the contest, the threads started by posters provided fodder for discussion. Second, the IBM summer interns were colocated and faced similar problems. They used the discus- 
sion forums to help each other, to share experiences and interests, to find roommates or to organize activities outside of work. In contrast, CHIplace participants were highly discretionary users with only common interests in human-computer interaction and the $\mathrm{CHI}$ conference. The introductions of paper preview and new CHIplace sections (HCI \& Culture and HCI Techniques) with articles from CHIplace members prompted more discussions over the last 11 weeks.

\section{Similarities in Usage of the Two Sites}

While the usage patterns for the two sites look very different at first glance, they actually have much in common. On both sites, most transitions stay within the same category or go from the home page to one of the categories. When removing the discussion forum usage that dominates Portkey and the CHIplace community and preview content, the usage distribution for the two sites is very similar with the people category dominating the usage. The transitions among the remaining categories are also very similar. This similarity might indicate that the users of the two different sites used interaction elements in similar fashions and frequencies. It also shows that the users of both sites were very interested in finding out about other people on the site.

The proportion of usage of the different categories on CHIplace stayed fairly constant over time. The discussion and community categories had the most variable usage depending on the addition of new community content such as stories and organization overviews and occasional increases in discussion activities. The conference preview activities (mostly the paper preview) started in the last 15 weeks and contributed $21 \%$ of the usage during the last 11 weeks.

\section{New CHIplace Members}

Another temporal trend concerns the addition of new CHIplace members (see Figure 10). After a fairly fast increase over the first two months, the rate of increase slowed down for the next eight months. With the start of the paper preview, we see a much faster increase, presumably because the full versions of the previewed papers are only accessible for CHIplace members and possibly because of the approach of the CHI 2002 conference.

\section{LESSONS LEARNED}

The paper examined the design of the Web sites in terms of the social functions of the infrastructure components. However, the underlying computational and content management components were very important to reuse and to a low maintenance and development effort. The use of dynamically generated pages from templates and a database simpli-

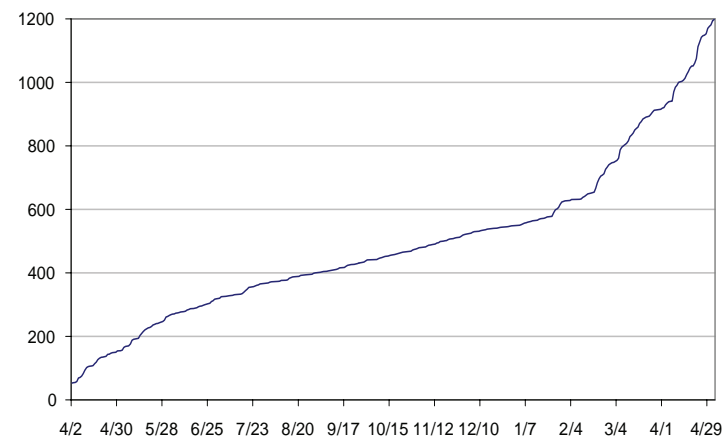

Figure 10: Increase of CHlplace Members. fied adding and modifying pages and creating custom information for signed-in users.

A supportive infrastructure alone is insufficient to attract and retain user interests. New and frequent content additions, be it editorial or user contributions, must occur or else user interest disappears quickly. Two other Web sites using our infrastructure, CHIplace 2003 and CSCWplace, provide little new editorial content and have little activity in the discussion forums. Perhaps, as a consequence, new users are registering at a slow rate.

In the absence of a large editorial staff, user and community contributions such as organizational overviews or articles on HCI topics provided an alternative source for editorial content on CHIplace. Portkey was more successful in fostering discussions because, in part, the Portkey members had more interests and problems in common. As well, the contest on Portkey helped to motivate a few to initiate some of the discussions that provided a critical mass of messages for others to respond to. Using moderators in the CHIplace paper preview turned out to be less effective. Lightweight social interaction tools such as quick polls provide lower barriers to participation and produces valuable user contributions.

Our usage analysis shows that information about other people, discussions, and community content is of great interest to the users. People pages were especially popular on both sites. Some users kept reloading the page to obtain new random picture galleries. Making people and their contributions visible is invaluable in capturing visitor interest.

Other than new content and discussion activity, email announcements were good means for drawing in new users and for renewing interest of existing users. Our data shows that such notifications caused spikes in the usage. Interesting members-only areas were also useful in encouraging visitors to register as members (see for example the increase in Figure 10 with the start of the paper preview in February).

\section{CONCLUSION}

The opportunities created by the Web open the doors to a broad range of interactions, some involving repeated interactions and some bringing strangers together. They can be brought to bear on many application domains where there is a need for human-to-human interaction mediated by sociotechnical elements, such as dynamic e-Business, customer relationship management, support networks, and electronic marketplaces [1]. Social interaction environments play an important role in the interaction and task experiences in these opportunities by coupling critical social relations with communication and information technologies [21].

In this paper, we presented a case study of the design of two social interaction Web sites: CHIplace and Portkey. We described five key design challenges that influenced the development of the Web sites, drawn from several related efforts to provide principles for the design of social interaction environments. We presented examples of how these challenges were addressed with socio-technical solutions. We analyzed usage patterns to examine the effectiveness of the designs for facilitating social interactions. Our results demonstrate that social interaction Web sites are effective in sustaining interest and in fostering social interactions. 


\section{REFERENCES}

1. A. Armstrong and J. Hagel III, The Real Value of Online Communities. Harvard Business Review, MayJune, pp. 134-141, 1996.

2. R. Axelrod. The Evolution of Cooperation. New York: Basic Books, 1984.

3. A. Bruckman. MOOSE Crossing: Construction, Community, and Learning in a Networked Virtual World for Kids, MIT Ph.D. Thesis, 1997.

4. D. Constant, L. Sproull, and S. Kiesler. The Kindness of Strangers: On the Usefulness of Electronic Weak Ties for Technical Advice. In S. Kiesler (ed.), Culture of the Internet, Lawrence Erlbaum Associates, New Jersey, pp. 303-322, 1997.

5. S. Farnham, L. Cheng, L. Stone, M. Zner-Godsey, C. Hibbeln, K. Syrjala, A.M. Clark, J. Abrams. HutchWorld: Clinical Study of Computer-Mediated Social Support for Cancer Patients and their Caregivers. In Human Factors in Computing Systems, CHI 2002 Conference Proceedings, ACM, New York, pp. 375-382, 2000.

6. K.E. Finn, A.J. Sellen, and S.B. Wilbur (eds.), VideoMediated Communication, Lawrence Erlbaum Associates, New Jersey, 1993.

7. G. Fischer, J. Grudin, R. McCall, J. Ostwald, D. Redmiles, B. Reeves, F. Shipman. Seeding, Evolutionary Growth and Reseeding: The Incremental Development of Collaborative Design Environments. In G. Olson, T. Malone, and J. Smith (eds.), Coordination Theory and Collaboration Technology, Lawrence Erlbaum Associates, New Jersey, pp. 447-472, 2001.

8. S. Harrison and P. Dourish. Re-Place-ing Space: The Roles of Place and Space in Collaborative Systems. In Proceedings of the Conference on Computer-Supported Cooperative Work (CSCW'96), ACM, New York, pp. 67-76, 1996.

9. T. Ishida and K. Isbister (Eds.). Digital Cities: Technologies, Experiences, and Future Perspectives. Lecture Notes in Computer Science. Springer, Berlin, 2000.

10. S.U. Kelly, C. Sung, and S. Farnham. Designing for Improved Social Responsibility, User Participation, and Content in On-Line Communities. In Human Factors in Computing Systems, CHI 2002 Conference Proceedings, ACM, New York, pp. 391 - 398, 2000.

11. A.J. Kim. Community Building on the Web. Peachpit Press, Berkeley, CA, 2000.

12. P. Kollock. Design Principles for Online Communities. Harvard Conference on the Internet and Society, 1996. Also available at: http://www.sscnet.ucla.edu/soc/ faculty/kollock/papers/design.htm.

13. P. Kollock. The Production of Trust in Online Markets. In E. J. Lawler, M. Macy, S. Thyne, and H. A. Walker (eds.), Advances in Group Processes, 16, JAI Press, Greenwich, CT, 1999. Also available at: http://www.sscnet.ucla.edu/soc/faculty/kollock/papers/ online_trust.htm.
14. R.E. Kraut, R.S. Fish, R.W. Root, and B.L. Chalfonte. Informal Communication in Organizations: Form, Function, and Technology. In S. Oskamp and S. Spacapan (eds.), People's Reactions to Technology: In Factories, Offices, and Aerospace, Sage Publication, pp. 145199, 1990.

15. A. Lee, C. Danis, T. Miller, and Y. Jung. Fostering Social Interaction in Online Spaces. In Proceedings of INTERACT 2001: IFIP TC.13 International Conference on Human-Computer Interaction, IOS Press, pp. 59-66, 2001.

16. K.D. Mickelson. Seeking Social Support: Parents in Electronic Support Groups. In S. Kiesler (ed.), Culture of the Internet. Lawrence Erlbaum Associates, New Jersey, pp. 157-178, 1997.

17. E.D. Mynatt, A. Adler, M. Ito, C., Linde and V.L. O'Day. The Network Communities of SeniorNet. In Proceedings of ECSCW'99, Kluwer Academic Publishers, London, pp. 219-238, 1999.

18. J.S. Olson, G.M. Olson, M. Storrøsten, and M. Carter. Groupwork up Close: A Comparison of the Group Design Process with and without a Simple Group Editor. ACM Transactions on Information Systems, 11(4), 321-348, 1993.

19. E. Ostrom. Governing the Commons: The Evolution of Institutions for Collective Action. Cambridge University Press, New York, 1990.

20. J. Preece. Online Communities: Designing Usability, Supporting Sociability. John Wiley \& Sons, Ltd., England, 2000.

21. P. Resnick. Beyond Bowling Together: SocioTechnical Capital. In J.M. Carroll (ed.), Human-Computer Interaction in the New Millennium, Addison-Wesley, New York, pp. 647-672, 2001.

22. H. Rheingold. The Virtual Community: Homesteading on the Electronic Frontier. pp. 1-16, 1993.

23. T.L. Roberts. Are Newsgroups Virtual Communities? In Human Factors in Computing Systems, CHI'98 Conference Proceedings, ACM, New York, pp. 360-367, 1998.

24. D. Schuler. HCI Meets the "Real World": Designing Technologies for Civic Use. In J.M. Carroll (ed.), Human-Computer Interaction in the New Millennium, Addison-Wesley, New York, pp. 627-646, 2001.

25. J. Short, E. Williams, B. Christie. The Social Psychology of Telecommunications. John Wiley \& Sons: New York, 1976.

26. L. Terveen and W. Hill. Beyond Recommender Systems: Helping People Help Each Other. In J.M. Carroll (ed.), Human-Computer Interaction in the New Millennium, Addison-Wesley, New York, pp. 487-509, 2000.

27. J.C. Thomas, W. Kellogg, T. Erickson. The Knowledge Management Puzzle: Human and Social Factors in Knowledge Management. IBM Systems Journal, 40(4), pp. 863-884, 2001. 